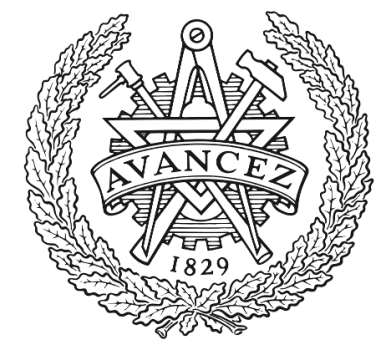

CHALMERS

UNIVERSITY OF TECHNOLOGY

\title{
Circulator Load Modulated Amplifier: A Non-Reciprocal Wideband and Efficient PA Architecture
}

Downloaded from: https://research.chalmers.se, 2023-04-26 12:13 UTC

Citation for the original published paper (version of record):

Zhou, H., Perez-Cisneros, J., Fager, C. (2021). Circulator Load Modulated Amplifier: A

Non-Reciprocal Wideband and Efficient PA Architecture. IEEE MTT-S International Microwave

Symposium Digest, 2021-June: 603-605. http://dx.doi.org/10.1109/IMS19712.2021.9574960

N.B. When citing this work, cite the original published paper. 


\title{
Circulator Load Modulated Amplifier: A Non-Reciprocal Wideband and Efficient PA Architecture
}

\author{
Han Zhou, Jose-Ramon Perez-Cisneros, Christian Fager \\ Chalmers University of Technology, SE-412 96 Gothenburg, Sweden \\ \{han.zhou, jospere, christian.fager\}@chalmers.se
}

\begin{abstract}
A novel power amplifier (PA) architecure, the Circulator Load Modulated Amplifier (CLMA), is presented and demonstrated. Based on a 3-port non-reciprocal combiner, the CLMA is able to modulate the load of a class-B amplifier by means of controlling the amplitude and phase of a class- $C$ amplifier. The architecture enables broadband highly-efficient operation over a reconfigurable output power control range. As a proof of concept, a narrowband prototype PA based on GaN transistors and a commercial circulator is employed to validate the CLMA concept. It exhibits peak output power of $43.1 \mathrm{dBm}$ and drain efficiency of $73 \%$ at 6-dB output power control range at a center frequency of $2.09 \mathrm{GHz}$.

Keywords - Circulator, power amplifier (PA), energy efficiency, wideband, load modulation, GaN, non-reciprocal.
\end{abstract}

\section{INTRODUCTION}

The increasing demand for larger capacity in recent and future wireless communication systems calls for broadband power amplifier (PA) architectures able to efficiently handle signals with high peak to average power ratio (PAPR).

Traditionally, efficiency enhancement techniques are based on dynamic load modulation and/or dynamic biasing concepts [1]. Among them, load modulating schemes have attracted increased interest because of the complexity and the bandwidth limitations inherent to the envelope tracking architecture [2]. However, the existing efficiency-bandwidth compromise in conventional load modulation based architectures, such as Doherty power amplifier (DPA) [3] or outphasing scheme [4], is a demanding challenge that PA designers need to address [5], [6].

Recently, the load modulated balanced amplifier (LMBA) has been introduced in [7] to enhance the bandwidth of conventional load modulated PAs. However, the circuit complexity of the LMBA is higher, the input and output quadrature couplers increase the loss, as well as the circuit size. Furthermore, the use of one extra transistor add cost.

In this paper, a novel active load modulation PA architecture, the Circulator Load Modulated Amplifier (CLMA), is proposed. The topology, which employs two active devices and a non-reciprocal combiner, is able to maintain high efficiency at both peak power and power back-off. Moreover, the availability of wideband low-loss circulators make this architecture promising for wideband applications. The CLMA has therefore potential to overcome many of the drawbacks of other architectures. In this work the CLMA concept is validated through a narrowband prototype design.

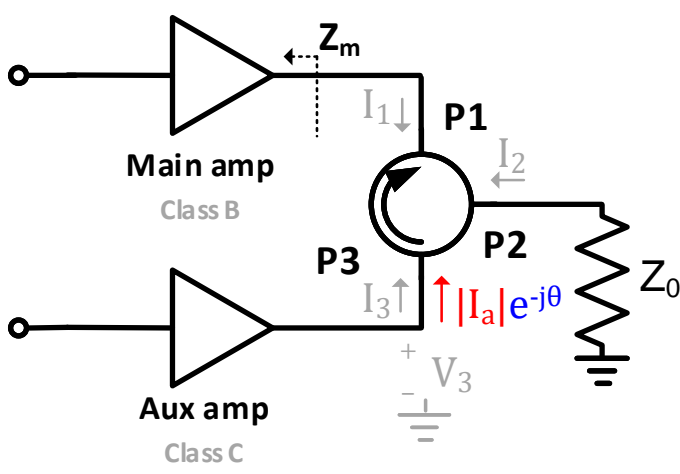

Fig. 1. Simplified block diagram of the ideal CLMA.

\section{THEORY}

Fig. 1 shows a simplified block-diagram of the proposed ideal CLMA architecture. It consists of a 3-port non-reciprocal combiner, a main class-B amplifier and an auxiliary class-C amplifier.

The CLMA operation is based on the fact that an auxiliary class-C amplifier is able to modulate the load presented to a main class-B amplifier by means of controlling the signal injected by the auxiliary amplifier through the non-reciprocal combiner. Moreover, the power injected by the main and auxiliary amplifiers can be fully delivered to the load, due to the inherent non-reciprocity property of the combiner network. This allows high efficiency to be maintained over a wide output power control range.

Eq. (1) presents the 3-port admittance parameter matrix for an ideal circulator with characteristic admittance $Y_{0}$. The port currents $I_{n}$ flow into the circulator whilst the voltages $V_{n}$ are measured with respect to a common ground connection (see Fig. 1).

$$
\left[\begin{array}{l}
I_{1} \\
I_{2} \\
I_{3}
\end{array}\right]=Y_{0}\left[\begin{array}{ccc}
0 & 1 & -1 \\
-1 & 0 & 1 \\
1 & -1 & 0
\end{array}\right]\left[\begin{array}{l}
V_{1} \\
V_{2} \\
V_{3}
\end{array}\right]
$$

As it can be seen in Fig. 1, the main and auxiliary amplifiers are connected to the circulator at ports P1 and $\mathrm{P} 3$, respectively. They have different amplitudes $I_{m}$ and $I_{a}$, respectively, with a phase offset $\theta$ between them. Thus, $I_{1}=$ $-I_{m}, I_{3}=-I_{a} e^{j \theta}$. The output port (P2) is terminated with a resistive load equal to the characteristic impedance of the circulator, $Z_{0}$. Therefore, $V_{2}=-Z_{0} I_{2}$. 


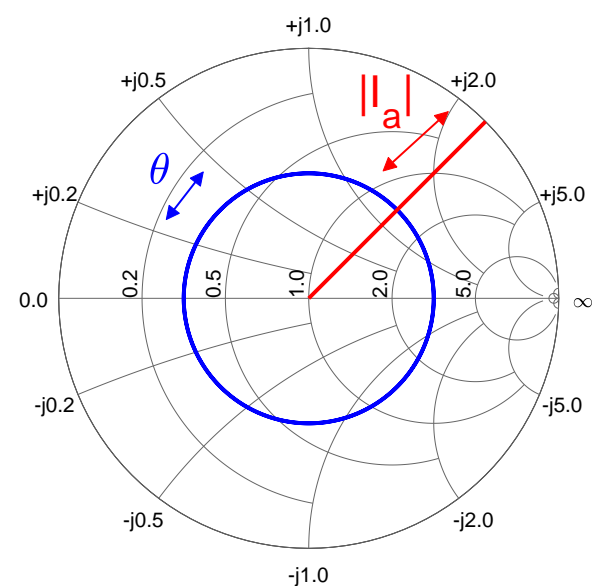

Fig. 2. Effect of auxiliary signal amplitude and phase variation on the impedance seen by the main amplifier of the CLMA architecture.

Substituting the above equations into the 3-port admittance matrix, the resulting set of equations can be solved to obtain the expression for the impedances seen by the main amplifier, denoted as $Z_{m}$, as follows

$$
Z_{m}=Z_{0}\left(1+2 \frac{I_{a} e^{j} \theta}{I_{m}}\right)
$$

Eq. (2) reveals that the impedance seen by the main amplifier can be dynamically modulated by varying the magnitude and phase of the auxiliary current. Fig. 2 shows the effect of signal amplitude and phase from the auxiliary amplifier on the impedance seen by the main amplifier.

Moreover, from the set of equations above, the relationship between the currents can be determined by

$$
I_{2}=I_{m}+I_{a} e^{j \theta} .
$$

Furthermore, it can be verified that $P_{2}=P_{m}+P_{a}$, which proves that the power injected by the main and auxiliary amplifiers is fully transferred to the output port. For simplicity, mathematical derivations are skipped.

According to the theory, it is possible to present the optimal impedance to the main amplifier at different output power levels by properly controlling the magnitude and phase of the auxiliary amplifier current. In addition, the power of both constituting amplifiers is delivered to the load. Hence, the CLMA architecture is capable of maintaining high efficiency over a large output power range. In principle, the bandwidth is limited only by the 3-port non-reciprocal output combiner.

It should be remarked that the impedance seen by the auxiliary amplifier is maintained constant, and equal to $Z_{0}$, due to the high isolation from main to auxiliary ports. Moreover, the main amplifier only needs to be matched to provide high efficiency at a single power level across the considered bandwidth. Therefore, the design requirements of the output matching networks of the constituting amplifiers of the CLMA are considerably relaxed compared to other load modulating architectures.

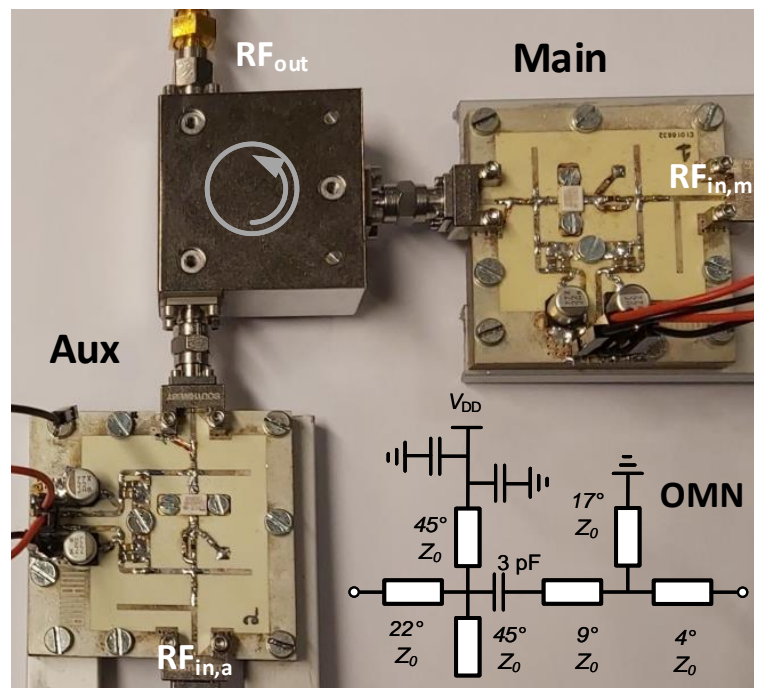

Fig. 3. Photograph of the CLMA demonstrator including detailed schematic of the OMN

\section{EXPERIMENTAL VERIFICATION}

As a proof of concept, a demonstrator is employed to validate the CLMA approach. It is based on GaN transistors and a commercial circulator.

A PA test board based on a 10-W GaN HEMT CGH40010F packaged transistor from Wolfspeed is used to perform as both main and auxiliary amplifiers. The output matching network $(\mathrm{OMN})$ of the PA test board was designed following a strategy for the maximization of back-off efficiency at $2.14 \mathrm{GHz}$.

The three-port SM2040C09 circulator from Quest Microwave is selected to act as the output combiner since it has both low insertion loss and high isolation.

Fig. 3 shows a photo of the CLMA demonstrator together with a detailed schematic of the OMN. In addition, a $3 \mathrm{~dB}$-Wilkinson power splitter and a phase shifter in the auxiliary amplifier branch were employed to characterize the CLMA in order to evaluate its performance.

The CLMA demonstrator has been characterized with static $\mathrm{CW}$ measurements. The drain bias of the main and auxiliary amplifiers were set to $28 \mathrm{~V}$ and $34 \mathrm{~V}$, respectively. The main amplifier was biased in class-B $(25 \mathrm{~mA}$ quiescent current) whilst the auxiliary amplifier was biased in class-C $\left(V_{G S}=-6 \mathrm{~V}\right)$. A input power splitting ratio of approximately $50 \%$ has been employed. The optimum phase delay for 1.99 , 2.04, 2.09 and $2.14 \mathrm{GHz}$ has been selected.

Fig. 4 shows the measured drain efficiency versus output power profiles for several frequencies. It exhibits of $43.3 \pm$ $0.4 \mathrm{dBm}$ and drain efficiency of $73.2-58 \%$ at 6-dB output power control range across $2.04-2.14 \mathrm{GHz}$. At $2.09 \mathrm{GHz}$, drain efficiency figures of $73 \% / 48 \%$ were measured over $6 / 10 \mathrm{~dB}$ of output power control range, respectively. Table I compares these results with those from representative state-of-the-art load modulated PAs.

\section{CONCLUSION}

A novel PA architecture based on a 3-port non-reciprocal combiner network has been proposed and verified. The use of 


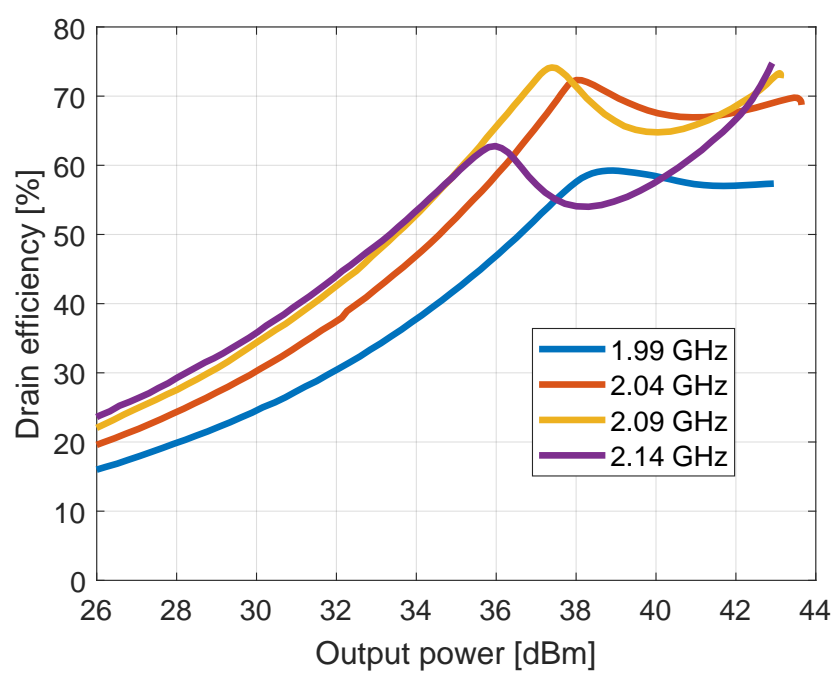

Fig. 4. Measured drain efficiency versus output power profiles at different frequencies.

Table 1. Comparison with state-of-the-art load modulated PAs.

\begin{tabular}{ccccc}
\hline Reference & Topology & Freq $(\mathrm{GHz})$ & $P_{\max }(\mathrm{dBm})$ & $\eta_{6 \mathrm{~dB} / 10 \mathrm{~dB}}(\%)$ \\
\hline$[8]$ & Outphasing & 0.7 & 47.4 & $80 / 70$ \\
\hline$[9]$ & Doherty & 1.95 & 44 & $62 / 52$ \\
\hline$[10]$ & Outphasing & 2.3 & 48.5 & $60 / 55$ \\
\hline$[11]$ & LMBA & 2.4 & 45.6 & $54 / 38$ \\
\hline$[12]$ & Doherty & 3.5 & 44.5 & $68 / 52$ \\
\hline This work & CLMA & 2.09 & 43.1 & $73 / 48$ \\
\hline
\end{tabular}

a non-reciprocal combiner reduces the interaction between the main- and auxiliary branches, which simplifies the design of the amplifiers. Combined with the availability of broadband low-loss circulators, it has the potential to provide broadband highly-efficient operation over large output power control range. As a first validation of the new PA architecture a narrowband high performance demonstrator has been realized.

\section{ACKNOWLEDGMENT}

This research has been carried out in the GigaHertz Centre in a joint project financed by the Swedish Government Agency for Innovation Systems (VINNOVA), Chalmers University of Technology, Ericsson, Gotmic, Infineon Technologies Austria, Qamcom, RISE, and SAAB.

\section{REFERENCES}

[1] F. H. Raab, P. Asbeck, S. Cripps, P. B. Kenington, Z. B. Popovic, N. Pothecary, J. F. Sevic, and N. O. Sokal, "Power amplifiers and transmitters for RF and microwave," IEEE Transactions on Microwave Theory and Techniques, vol. 50, no. 3, pp. 814-826, 2002.

[2] P. Asbeck and Z. Popovic, "ET Comes of Age: Envelope Tracking for Higher-Efficiency Power Amplifiers," IEEE Microwave Magazine, vol. 17 , no. 3, pp. 16-25, 2016.

[3] W. H. Doherty, "A New High Efficiency Power Amplifier for Modulated Waves," Proceedings of the Institute of Radio Engineers, vol. 24, no. 9, pp. 1163-1182, 1936.

[4] H. Chireix, "High power outphasing modulation," Proceedings of the Institute of Radio Engineers, vol. 23, no. 11, pp. 1370-1392, 1935.

[5] G. Nikandish, R. B. Staszewski, and A. Zhu, "Breaking the bandwidth limit: A review of broadband doherty power amplifier design for $5 \mathrm{~g}$," IEEE Microwave Magazine, vol. 21, no. 4, pp. 57-75, 2020.
[6] R. A. Beltran, "Broadband outphasing transmitter using class-e power amplifiers," in 2019 IEEE MTT-S International Microwave Symposium (IMS), 2019, pp. 67-70.

[7] D. J. Shepphard, J. Powell, and S. C. Cripps, "An efficient broadband reconfigurable power amplifier using active load modulation," IEEE Microw. Wireless Compon. Lett., vol. 26, no. 6, pp. 443-445, Jun. 2016.

[8] D. Vegas, M. Pampín, J. Perez-Cisneros, M. N. Ruiz, A. Mediavilla, and J. A. García, "Uhf class-e power amplifier design for wide range variable resistance operation," in 2018 IEEE/MTT-S International Microwave Symposium - IMS, 2018, pp. 297-300.

[9] M. Özen, K. Andersson, and C. Fager, "Symmetrical doherty power amplifier with extended efficiency range," IEEE Transactions on Microwave Theory and Techniques, vol. 64, no. 4, pp. 1273-1284, 2016.

[10] D. A. Calvillo-Cortes, M. P. van der Heijden, M. Acar, M. de Langen, R. Wesson, F. van Rijs, and L. C. N. de Vreede, "A package-integrated chireix outphasing rf switch-mode high-power amplifier," IEEE Transactions on Microwave Theory and Techniques, vol. 61, no. 10, pp. 3721-3732, 2013.

[11] P. H. Pednekar, W. Hallberg, C. Fager, and T. W. Barton, "Analysis and design of a doherty-like rf-input load modulated balanced amplifier," IEEE Transactions on Microwave Theory and Techniques, vol. 66, no. 12 , pp. 5322-5335, 2018.

[12] M. Özen and C. Fager, "Symmetrical doherty amplifier with high efficiency over large output power dynamic range," in 2014 IEEE MTT-S International Microwave Symposium (IMS2014), 2014, pp. 1-4. 